\title{
THE EIGHTEENTH SUMMER MEETING OF THE AMERICAN MATHEMATICAL SOCIETY.
}

THE eighteenth summer meeting of the Society was held at Vassar College on Tuesday and Wednesday, September 12-13, 1911, extending through two sessions on Tuesday and a morning session on Wednesday. The attendance included the following thirty-two members:

Dr. R. C. Archibald, Dr. F. W. Beal, Dr. Elizabeth R. Bennett, Professor G. D. Birkhoff, Professor Joseph Bowden, Professor W. G. Bullard, Dr. H. T. Burgess, Professor F. N. Cole, Professor L. L. Conant, Dr. Elizabeth B. Cowley, Mr. E. P. R. Duval, Professor L. P. Eisenhart, Professor T. S. Fiske, Mr. Meyer Gaba, Miss Ida Griffiths, Dr. W. A. Hurwitz, Professor C. J. Keyser, Professor W. R. Longley, Professor J. H. Maclagan-Wedderburn, Professor G. A. Miller, Dr. H. H. Mitchell, Professor G. D. Olds, Professor Arthur Ranum, Dr. J. E. Rowe, Professor C. H. Sisam, Professor A. W. Smith, Professor Virgil Snyder, Professor E. B. Van Vleck, Professor Oswald Veblen, Professor A. G. Webster, Professor H. S. White, Professor J. W. Young.

Ex-Presidents T. S. Fiske and H. S. White occupied the chair at the several sessions. The Council announced the election of the following persons to membership in the Society: Professor Frederick Anderegg, Oberlin College; Dr. C. E. Brooks, Northwestern University; Mr. G. G. Brower, Cascadilla School; Mr. W. C. Graustein, Harvard University; Dr. Dunham Jackson, Harvard University; Mr. W. V. Lovitt, University of Washington; Mr. J. C. Rayworth, Washington University; Mr. L. L. Smail, University of Washington; Dr. E. B. Stouffer, University of Illinois; Dr. S. E. Urner, University of Wisconsin; Professor J. N. Van der Vries, University of Kansas; Mr. C. W. Wester, University of Washington. Twelve applications for membership in the Society were received.

On both days of the meeting luncheon was served in the College buildings. On Tuesday evening twenty-nine of the members gathered at the customary informal dinner, at the close of which Professor Birkhoff spoke briefly on Moore's general analysis and Professor Webster on wider views in mathematics and physics. Wednesday afternoon was devoted 
to an excursion to Lake Mohonk. At the close of the meeting the hospitality of Vassar College was gratefully acknowledged by a vote of thanks.

During the summer the following committees have been appointed: on nomination of officers and members of the Council, Professors Bôcher, Dickson, and Morley; on arrangements for the summer meeting and colloquium at Madison in 1913, Professors Moore, Osgood, Van Vleck, and the Secretary.

The following papers were read at the summer meeting:

(1) Professor Edmund Landau: "Ueber eine idealtheoretische Funktion."

(2) Dr. W. A. Hurwitz: "On the pseudo-resolvent to the kernel of an integral equation."

(3) Dr. W. A. Hurwitz: "On mixed linear integral equations."

(4) Dr. Elizabeth R. Bennett: "Simply transitive primitive groups whose maximal subgroup contains a transitive constituent of order $p^{2}$ or $p q$, or a transitive constituent of degree 5."

(5) Professor Florian Cajori: "On a rare book of Michel Rolle and the history of 'Rolle's theorem.",

(6) Professor I. C. Karpinski: "The algebra of Abū Kāmil Shojā ben Aslam."

(7) Dr. F. W. Beal: "Normal congruences determined by centers of geodesic curvature."

(8) Professor ARNold Емch: "On the congruence of rays realizing circular transformations between two planes."

(9) Professor Joseph Bowden: "The two fundamental relations of the calculus" (preliminary communication).

(10) Dr. J. E. Rowe: "Covariant curves of the $R^{4}$ and $R^{5}$."

(11) Professor G. A. Miller: "A third generalization of the groups of the regular polyhedra."

(12) Professor G. A. Miller: "Some properties of the group of isomorphisms."

(13) Professor L. P. Eisenhart: "Minimal surfaces in plane four-space."

(14) Professor Arthur Ranum: "On the projective differential geometry of spreads generated by $\infty^{1}$ flats."

(15) Mr. E. W. CAstle: "A graduation of the combined experience table of mortality to Makeham's formula by the method of moments."

(16) Dr. S. Lefschetz: "On the existence of loci with given singularities." 
(17) Dr. S. Lefschetz: "On some topological properties of plane curves."

(18) Professor Virgil SNyder: "Periodic quadratic transformations in a ternary field."

(19) Professor A. G. Webster: "On a new mixed boundary problem in connection with the telegrapher's equation."

(20) Professor A. G. Webster: "On the wave potential of a circular ring of sources."

(21) Professor A. G. Webster: "Solid viscosity versus elastic hysteresis in the transverse vibration of an elastic bar."

(22) Professor G. D. BIRKногF: "New proof of a theorem concerning matrices of analytic functions."

(23) Professor G. D. Birkhoff: "On the simplest type of irregular singular point."

(24) Professor G. A. BuIss: "A generalization of the preparation theorem for a power series in several variables."

(25) Professor Oswald Veblen: "On the definition of multiplication of irrational numbers."

(26) Dr. H. T. BuRgEss: "One-parameter groups of contact transformations defined on a fixed quadric by a bilinear form."

(27) Professor Joseph Bowden: "Making a recitation schedule."

Mr. Castle's paper was communicated to the Society by Professor Glover. Dr. Lefschetz was introduced by Professor Webster. The papers of Professors Landau, Cajori, Karpinski, Emch, Miller (second paper), Mr. Castle, and Professor Bliss were read by title. Professor Landau's paper will appear in the October number of the Transactions. Abstracts of the other papers follow below. The abstracts are numbered to correspond to the titles in the list above.

2. The method developed by Fredholm for the solution of the linear integral equation is comparatively simple in case the homogeneous equation admits no solutions not identically zero; a resolvent function to the kernel of the equation can be expressed in terms of the determinant and the first minor. If the homogeneous equation admits non-zero solutions, the development of necessary and sufficient conditions for the existence of solutions of the non-homogeneous equation by Fredholm's method, while similar in character to the simpler case, involves the introduction of minors of higher order, thereby necessitating somewhat tedious and complicated algebraic manipulation. 
For the sake of simplicity of presentation, it seems valuable to secure these results more simply. Dr. Hurwitz's first paper, by the artifice of transforming a given integral equation into a new one for which the corresponding homogeneous equation has no solutions other than zero, proves, without the introduction of minors of higher order, the following theorems, furnishing the theory desired:

1) If the kernel $K(x, y)$ is continuous, the equations

$$
\begin{aligned}
& \varphi(x)=\int_{a}^{b} K(x, s) \varphi(s) d s, \\
& \psi(x)=\int_{a}^{b} \psi(s) K(s, x) d s
\end{aligned}
$$

have each a finite number of linearly independent continuous solutions, and this number is the same for the two equations.

2) If the complete sets of normal orthogonal continuous solutions of (1), (1') are respectively $\varphi_{1}(x), \varphi_{2}(x), \ldots, \varphi_{m}(x) ; \psi_{1}(x)$, $\psi_{2}(x), \ldots, \psi_{m}(x)$, there exists a continuous function $k(x, y)$, the pseudo-resolvent to the given kernel $K(x, y)$, such that

$$
\begin{aligned}
& k(x, y)=K(x, y)+\int_{a}^{b} K(x, s) k(s, y) d s-\sum_{i=1}^{m} \psi_{i}(x) \psi_{i}(y), \\
& k(x, y)=K(x, y)+\int_{a}^{b} k(x, s) K(s, y) d s-\sum_{i=1}^{m} \varphi_{i}(x) \varphi_{i}(y) .
\end{aligned}
$$

3) A necessary and sufficient condition that the equation

$$
u(x)=f(x)+\int_{a}^{b} K(x, s) u(s) d s
$$

(where $f(x)$ is continuous) have a continuous solution, is that

$$
\int_{a}^{b} \psi_{i}(x) f(x) d x=0 \quad(i=1,2, \ldots, m) .
$$

If this condition is fulfilled, the most general solution of (2) is

$$
u(x)=f(x)+\int_{a}^{b} k(x, s) f(s) d s+\sum_{i=1}^{m} c_{i} \varphi_{i}(x),
$$

where $c_{1}, c_{2}, \ldots, c_{m}$ are constants. 
3. By a mixed integral equation is understood one which involves the unknown function under several integral signs, operating over regions of different numbers of dimensions, and which also involves the value of the unknown function at isolated points. Dr. Hurwitz's second paper seeks to indicate how the theory of mixed linear integral equations follows the laws governing linear equations in general, by the establishment of the following theorem, bearing on an especially simple case:

Let $f(x), k_{1}(x), k_{2}(x), \cdots, k_{m}(x)$ be continuous functions of $x$, $a \leqq x \leqq b$, and $K(x, y)$ a continuous function of $x$ and $y$, $a \leqq x \leqq b, a \leqq y \leqq b$; let $x_{1}, x_{2}, \cdots, x_{m}$ lie in the interval $a \leqq x$ $\leqq b$. Then if the homogeneous mixed linear integral equation

$$
u(x)=\sum_{i=1}^{m} k_{i}(x) u\left(x_{i}\right)+\int_{a}^{b} K(x, s) u(s) d s
$$

has no continuous solution, $a \leqq x \leqq b$, other than $u(x) \equiv 0$, the equation

$$
u(x)=f(x)+\sum_{i=1}^{m} k_{i}(x) u\left(x_{i}\right)+\int_{a}^{b} K(x, s) u(s) d s
$$

has one and only one continuous solution.

4. Certain restrictions on the transitive constituents occurring in the maximal subgroup of a simply transitive primitive group when the maximal subgroup contains a transitive constituent of degree 3 or 4 or a transitive constituent of order $p$, have been determined. Dr. Bennett considers how the degree of a simply transitive primitive group and the transitive constituents in its maximal subgroup are limited when the maximal subgroup contains a transitive constituent of order $p^{2}$ or $p q$ or a transitive constituent of degree 5 .

5. Professor Cajori's paper describes Rolle's Démonstration d'une méthode pour résoudre les égalitez de tous les degrez, etc., a book which has not been previously described by historians. Recent doubts as to whether "Rolle's theorem" is due to Rolle are removed by this book, which gives the theorem in the course of the demonstration of Rolle's "method of cascades." The book gives also the solution of equations by the intersections of circles and parabolas, and a discussion of quadratic residues containing proofs of the theorem of Diophantus 
that no integer of the form $4 n+3$ can be the sum of two squares and of the theorem that no integer $8 n+7$ can be the sum of three squares. "Rolle's theorem" apparently was not named after Rolle until after the middle of the nineteenth century. It rose to prominence after $\mathrm{O}$. Bonnet based upon it a proof of the theorem of mean value (Serret, Calcul differentiel et intégral, 1868, page 17) and Taylor's theorem came to be proved by the mean value theorem (Homersham-Cox and Rouché).

6. In 1841 Chasles called attention to the Latin translation of an Arabic Algebra which is found in the Paris manuscript 7377A. This Algebra is now known to be the work of Abu Kāmil Shojā ben Aslam, who flourished during the early tenth century. Two commentaries on this Algebra were written within the same century and an Arabic historian, Ibn Khaldūn (1332-1406) states that a Spanish commentary was written by one Al-Korekhi, otherwise unknown. Mordechai Finzi (c. 1460) of Mantua made a Hebrew translation which bears internal evidence that it was based upon a Spanish version. Up to the present time copies have been found of the Latin and Hebrew translations only.

Professor Karpinski presents the first study which has been made of this Algebra since Chasles called attention to it. This shows that Abū Kāmil devised several new geometrical solutions of the three types of quadratic equations $\left(x^{2}+b x=c\right.$, $\left.x^{2}+c=b x, x^{2}=b x+c\right)$ but that otherwise he followed. closely the lines laid down by Al-Khowarizmi and that Leonard of Pisa drew many of his algebra problems, complete with their solutions, from $A b \bar{u} \bar{u}$ Kàmil.

7. In 1892 Caronnet announced the following theorem: The necessary and sufficient condition that the lines which join the centers of geodesic curvature of the curves of an orthogonal system on a surface shall form a normal congruence is that the corresponding radii of geodesic curvature be functions of one another or that the curves in one family or both have constant geodesic curvature. Dr. Beal's paper is concerned with the determination of normal congruences of this kind and with an investigation of their properties. For the sake of brevity he has referred to them as associated normal congruences.

When both radii of geodesic curvature are constant the as- 
sociated normal congruence is the congruence of normals of a Bianchi transform of the given surface. In this case the given surface must be pseudospherical and of total curvature equal to the negative of the sum of the squares of the constant geodesic curvatures. Associated normal congruences exist for any surface for any given functional relation between the radii of geodesic curvature and are determined by a partial differential equation of the first order, which is also of the first degree when and only when one radius is equal to a constant or to a constant times the other. The condition in the theorem due to Caronnet that the system of curves be orthogonal may be replaced by the condition that the curves of the two families intersect under a constant angle. The congruences determined by these systems will be associated normal congruences.

8. As is well known, the Steinerian transformation realizes in a very simple geometric manner a general uniform quadratic transformation between two planes. This correspondence is obtained by the intersection of the congruence of rays through any two skew lines with those planes. The special case of circular transformations is obtained by assuming in a definite manner two of the fundamental points in each plane as the circular points at infinity. In this case the construction in the Steinerian sense becomes illusory. It is however possible to establish such a congruence by means of two inversions in space and it is the purpose of Professor Emch's paper to investigate the properties of this congruence and its limiting surface, which is of the fourth order.

9. In order to give a clear understanding of the fundamental theorems of the integral calculus Professor Bowden finds it useful to employ the symbols $D$ and $I$ respectively for the relations "is the derivative of" and "is an integral of." Thus $2 x D x^{2}, x^{2}+4 I 2 x,(u I v)=(v D u)$. Each of these relations is the converse of the other. Each is non-reflexive, non-symmetric, non-transitive. Among the fundamental laws are: $(u D v) \supset(-u D-v),(u D v) \frown(w D s) \supset(u+w) D(v+s),(u D v) \frown$ $(w D s) \supset(u s+v w) D(v s), \quad(u I v) \supset(-u I-v), \quad(u I v) \frown(w I s)$ $\supset(u+w) I(v+s),(u I v) \frown(w I s) \supset u w I(v w+u s)$.

10. In his dissertation, which appeared in the July Transactions, Dr. Rowe has considered certain covariant curves of the 
$R^{4}$ which are combinants of two binary quartics cut out by two secant lines of the $R^{4}$; these curves are expressible in terms of three-rowed determinants of the type $|a b x|$ and are called combinant curves of the $R^{4}$. In Section I of the present paper certain covariant loci of the $R^{4}$ are obtained which are expressible in terms of three-rowed determinants of the types $|a b c|$ and $|a b x|$; these are not combinant curves as defined above and for convenience are called bicombinant curves of the $R^{4}$.

The conic $A-16 B=0$ (conic on flexes of $R^{4}$ ) cuts out two other points besides the flexes which we call $q_{i}$; these two points determine a line $L_{4}$ which determines two other points $r_{i}$. $L_{4}$ is defined as a member of a pencil of bicombinant lines as follows:

$$
5 L_{4}=L_{3}+4\left(Q_{1}^{2}-4 Q_{0} Q_{2}\right) L_{1}
$$

where $L_{1}$ cuts out the two $q_{i}$ on $A-12 B=0$ (envelope of flex lines of cubic osculants of the $R^{4}$ ) and is the locus such that tangents to the $R^{4}$ from it have their parameters apolar to the flexes; the expression in the parenthesis of (1) is the condition for the $q_{i}$ to unite; and $L_{s}$ is a locus such that tangents from it to $R^{4}$ have their parameters apolar to the $q_{i}$ each taken three times. The equation of the line $L_{4}$ and the quadratic giving the $r_{i}$ are derived in this way. The condition that the $q_{i}$ and $r_{i}$ be apolar is the discriminant of the conic $A-16 B=0$.

Two bicombinant conics are given: the conic on the nodes and $q_{i}$, and the conic on the nodes and $r_{i}$.

The bicombinant cubic on the flexes and nodes of the $R^{4}$ is written out in full. By mere substitutions in this equation are obtained the following bicombinant cubics: on nodes and $q_{i}$ each taken three times; on nodes, $q_{i}$ each taken twice, and on $r_{i}$; on nodes, $q_{i}$, and on $r_{i}$ each taken twice; on nodes, and $r_{i}$ each taken three times.

In Section II the combinant curves of the $R^{5}$ of orders two and three are considered. The $R^{5}$ has a pencil of combinant conics having double contact on the covariant line. The combinant cubics of the $R^{5}$ are expressed in terms of four among which no linear relation exists in general. There is a unique combinant cubic expressed in terms of the above four which cuts out the flexes of the $R^{5}$ and six other points.

Also, a method is given for finding a certain set of invariants for any rational curve of odd order by reason of the $R^{2 n+1}$ having a covariant rational line quartic whose invariants are known. These invariants are actually found for the $R^{5}$. 
11. The abstract properties of the regular polyhedron groups were first developed by Sir W. R. Hamilton in a paper presented to the Royal Irish Academy in 1856. These groups are defined by three equations between the two generators $s_{1}, s_{2}$. The equations may be written in the form

$$
s_{1}^{a}=s_{2}^{\beta}=\left(s_{1} s_{2}\right)^{\gamma}=1,
$$

where two of the three positive integers $\alpha, \beta, \gamma$ are equal to 2 and 3 , while the third has the value 3,4 , or 5 , according as the group is tetrahedral, octahedral, or icosahedral, respectively. In the present paper Professor Miller considers the possible groups when the last condition is omitted. That is, he considers the groups generated by $s_{1}, s_{2}$ when these operators satisfy two conditions of the form

$$
s_{1}^{a}=s_{2}^{\beta}=\left(s_{1} s_{2}\right)^{\gamma}
$$

with the same restrictions on $\alpha, \beta$, and $\gamma$ as before.

It is proved that no more than four non-abelian groups can satisfy such a pair of conditions, and that the present generalizations lead to simpler systems of groups than the two earlier generalizations considered by the same author. The following theorems were proved: If two non-commutative operators satisfy the conditions $s_{1}^{3}=s_{2}^{3}=\left(s_{1} s_{2}\right)^{2}$, they generate either the tetrahedral group or the non-twelve group of order 24; if they satisfy the conditions $s_{1}^{3}=s_{2}^{4}=\left(s_{1} s_{2}\right)^{2}$, they generate either the octahedral group or a group of order 48 known as $G_{52}$; if they satisfy the two conditions $s_{1}^{3}=s_{2}^{5}=\left(s_{1} s_{2}\right)^{2}$, they generate either the icosahedral group or a group of order 120 known as $G_{120}$. In all the other possible cases the non-abelian groups are either the direct products of one of the given groups and a cyclic group or an isomorphism between such groups.

12. The first part of Professor Miller's second paper is devoted to a study of the group of isomorphisms $I$ of an abelian group $G$. It is pointed out that $I$ can always be represented as a transitive substitution group on letters corresponding to a set of operators of $G$ and that this can be done in only one way. When $I$ is represented as such a substitution group it is very easy to prove that unless $G$ is of order $2^{m}$ and of type $(1,1,1$, ...) its group of isomorphisms cannot be represented as a substitution group on letters corresponding to a set of subgroups of $G$. 
The principal theorems proved in the second part of this paper may be stated as follows: The group of isomorphisms of a non-abelian group cannot be represented transitively on letters corresponding to a set of commutative operators of this non-abelian group. If the group of isomorphisms $I$ of a group $G$ is represented on letters corresponding to a set of operators of $G$, then $I$ cannot be more than doubly transitive unless it is the symmetric group of order 6 . If $I$ is doubly transitive, and is not this symmetric group, then the subgroup composed of all the substitutions of $I$ which omit one letter is always imprimitive.

13. At the April meeting of the Society Professor Eisenhart established a method of giving to the parametric equations of any minimal curve in plane four-space a form analogous to the Weierstrass equations of minimal curves in ordinary space. In the present paper he makes use of these forms in order to define and discuss minimal surfaces (dimensional spreads) in plane four-space. This form is of particular value in the study of algebraic surfaces. The geometry of a general minimal surface in the neighborhood of a point is studied to advantage. With any such minimal surface it is possible to associate a family of applicable surfaces analogous to the group of associate minimal surfaces in ordinary space and therefrom follow formulas analogous to the formulas of Schwarz, with the consequent theory concerning the determination of a minimal surface by a strip. Reference is made to the minimal surfaces in four-space, defined by an equation of the form $z+i t=f(x+i y)$, where $f$ is an analytic function; these surfaces have been considered from another point of view by Karl Kommerell under the title Riemann Surfaces. This paper will be published in the American Journal of Mathematics.

14. In this paper Professor Ranum generalizes to $n$-dimensional space the results of his paper on "Ruled surfaces and planed hypersurfaces in four-dimensional space," of which an abstract is given in the BuLleTIN, volume 17, page 302. An $m$-dimensional spread $S_{m}$ generated by $\infty^{1}$ flats (linear spaces) is given by a set of homogeneous parametric equations. Its tangent spread $S_{m+r}$, is defined as the locus of the connecting flats of its consecutive generators. The focal spread of $S_{m}$, namely the locus of the flats of intersection of its consecutive 
generators, is shown to be an $S_{m-r-r}$. The number $r$ is called the rank of $S_{m} . S_{m+r}$ and $S_{m-r}$ are in general of lower rank than $S_{m}$. The focal spread of the tangent spread of $S_{m}$ is in general a larger spread enclosing $S_{m}$. Calling the focal spread of the focal spread the 2 nd focal spread, etc., the author shows that the 1st, $2 \mathrm{nd}, \ldots$, . ith focal spreads of the $i$ th tangent spread of $S_{m}$ are all of the same rank and all enclose $S_{m}$. Various other projective differential properties of these spreads are derived, the principle of duality is employed, and a general scheme is obtained for classifying the spreads.

15. The chief purpose of Mr. Castle's paper is to present a graduation of the combined experience of actuaries table of mortality to Makeham's formula by the method of moments. The different methods of graduating a mortality table may be roughly classified under two heads: those in which some formula is assumed to express the functional relation between the number living and the age $x$, the arbitrary constants involved being determined from the original data; and, second, those in which the number living at any age $x$ is derived from the original data in such a way as to produce a smooth curve without satisfying any functional relation.

In the graduation here presented Makeham's formula

$$
y=k s^{x} g^{c^{x}}
$$

was assumed to express the functional relation between the number living $y$ and the age $x$, the arbitrary constants $k, s, g$, and $c$ being determined by the method of moments. Briefly, the theory involved in graduation by the method of moments is that in fitting an assumed formula to statistical data the values of the constants which depend upon all the given data are obtained by finding the area and moments of the theoretical curve, and equating them to the corresponding area and moments as obtained from the statistical data.

The work has been carried out in considerable detail with the secondary object of being of use to those who desire to employ the method of moments in graduating similar statistical data. The paper will be published in the Annals of Mathematics.

16. The main point of Dr. Lefschetz's first paper in the introduction of a new inequality which the Plückerian numbers of any 
algebraic multiplicity have to satisfy. Plane curves are chiefly treated. Adopting Salmon's notations, since a double point requires one condition, a cusp two conditions to be satisfied by the coefficients of the general curve of a given order $m$, we must have

$$
\delta+2 k \leqq \frac{m(m+3)}{2}-8
$$

for such conditions are expressed evidently by the vanishing of as many invariants. A discussion follows, and maxima are given for the number of cusps alone. The question of the maximum of cusps for a given deficiency is also treated. Finally the same method is applied to surfaces. The cases of surfaces of the third, fourth, and fifth orders are treated at some length, and for them maxima for the number of binodes found. To mention one interesting result, it is found that a surface $S_{4}$ can have no binodes if it has more than 14 nodes, and never more than 8 binodes.

17. The chief feature of Dr. Lefschetz's second paper is an application of inversion to a descriptive study of curves. First, some well known properties of open or closed branches receive new proofs. Next a study of curvature follows with an intended application to inflexions of certain branches by means of an inversion. Then a detailed study is made of closed branches having one single multiple point of multiplicity $k$. A representation of such a system is given by means of a sketch, the chief feature of which is the formation of certain polygons having for vertices those of a regular polygon of $k$ sides. By means of some simple properties of such polygons, a theorem is proved for the case of $k=2 n+1$, which is then shown to be equivalent to the theorem of Möbius giving 3 as the minimum of inflexions of an odd branch without any multiple points. Really the idea is to substitute for a curve having infinite branches one lying at finite distance. Möbius did this by projecting plane curves on a sphere; the author obtains the same results by inverting the curve with respect to a pole through which it does not pass.

18. In the study of finite groups of Cremona transformations extensive use is made of the composition of each generator in terms of quadratic transformations. If two fundamental points are identical with two fundamental points of the inverse 
system the iteration of the quadratic transformation is also quadratic (or the identity). Starting from the theorem that in a cyclic collineation a point and its successive images lie on a conic, Kantor drew similar conclusions concerning quadratic transformations. Professor Snyder showed that neither one of these theorems is true unless restrictions are imposed on the coefficients, and that in consequence extensive categories of real transformations are omitted in Kantor's classification.*

19. If $u$ and $v$ stand for the current and potential respectively multiplied by an exponential factor, each satisfies the equation

$$
\frac{\partial^{2} u}{\partial^{2} x}-\frac{\partial^{2} u}{\partial y^{2}}+u=0
$$

and the problem treated in Professor Webster's first paper is the satisfaction of Cauchy's conditions, function and conormal derivative being given for $y=0, x \geqq 0$, while for $x=0, y \geqq 0$ the condition is

$$
\begin{aligned}
a_{0} u & +a_{1} \frac{\partial u}{\partial y}+a_{2} \frac{\partial^{2} u}{\partial y^{2}}+\ldots+a_{n} \frac{\partial^{n} u}{\partial y^{n}} \\
& +b_{0} v+b_{1} \frac{\partial v}{\partial y}+b_{2} \frac{\partial^{2} v}{\partial y^{2}}+\ldots+b_{m} \frac{\partial^{m} v}{\partial y^{m}}=\psi(y) .
\end{aligned}
$$

By means of Riemann's method, integration around the proper contour leads to a Volterra integro-differential equation for the conormal derivative, which gives the solution by the usual formula.

20. In Professor Webster's second paper the solution of the wave equation is found in the form of a definite integral, which is developed into two series for practical computation.

21. In Professor Webster's third paper the theory of the normal vibrations of a bar vibrating transversely is worked out according to Voigt's theory of viscosity, and also according to the theory of heredity as developed by Boltzmann, Wiechert, and especially Volterra, and the results contrasted. Experiments favor the heredity theory.

*S. Kantor, "Premiers fondements pour une théorie des transformations périodiques univoques," Atti della R. Accademia . . . di Napoli, ser. 2, vols. 3 and 4 (1891), pp. 1-356. 
22. In this note Professor Birkhoff employs the theory of integral equations to derive another proof of a theorem which was stated by him in an earlier paper on singular points of ordinary linear differential equations (Transactions of the Society, volume 4 (1908), pages 436-470).

23. In a second note Professor Birkhoff makes a detailed study of the irregular singular point of rank zero for a linear differential equation of the second order. The method depends on the theorem of the previous note.

24. The paper of Professor Bliss contains a generalization of a well-known theorem concerning a power series in several variables $F\left(x_{1}, x_{2}, \cdots, x_{m} ; y\right)$. This theorem states that if $F(0,0, \cdots$, $0 ; y)$ begins with a term of degree $v$ in $y$, then there exists a unique series $M\left(x_{1}, x_{2}, \cdots, x_{m} ; y\right)$ with constant term different from zero, such that the product $M F$ is a polynomial in $y$ of the form

$$
M F=y^{v}+a_{1} y^{v-1}+a_{2} y^{v-2}+\cdots+a_{v-1} y+a_{v},
$$

where the coefficients $a_{l}$ are power series in $x_{1}, x_{2}, \cdots, x_{m}$ which have no constant terms. Evidently in a sufficiently small region about the origin the roots of the function $F$ are all roots of the polynomial, and vice versa.

The generalization has to do with a system $f$ functions $F_{a}\left(x_{1}, x_{2}, \cdots, x_{m} ; y_{1}, y_{2}, \cdots, y_{n}\right)(a=1,2, \cdots, n)$ for which the series $F_{a}\left(0,0, \cdots, 0 ; y_{1}, y_{2}, \cdots, y_{n}\right)$ begin with so-called "characteristic" polynomials $f_{a}\left(y_{1}, y_{2}, \cdots, y_{n}\right)$, homogeneous and of degrees $v_{a}$, respectively. Under certain restrictions on the coefficients of the functions $f_{a}$, there will then always exist a polynomial

$$
P\left(x_{1}, x_{2}, \cdots, x_{m} ; y_{n}\right)=y_{n}^{N}+a_{1} y_{n}^{N-1}+\cdots+a_{N-1} y+a_{N}
$$

of degree $N=v_{1} v_{2} \cdots v_{n}$, such that every root of the equations $F_{a}=0$ in a sufficiently small region about the origin makes the polynomial vanish, and conversely to every root of the polynomial there corresponds a root of the equations. The polynomial is linearly expressible in the form

$$
P=M_{1} F_{1}+M_{2} F_{2}+\cdots+M_{n} F_{n},
$$

where the coefficients $M_{a}$ are series in $x_{1}, x_{2}, \cdots, x_{m} ; y_{1}, y_{2}, \cdots, y_{n}$. 
25. In the genetic theory of the irrational numbers, each open cut in the scale of rationals determines an irrational. Arbitrary definitions are given of the operations of addition and multiplication, which are then proved to satisfy the same laws of combination (associative, distributive, commutative, inverse) as the rational numbers. Professor Veblen showed that it is possible, without altering the definition of addition, to give another definition of multiplication such that all the laws of combination hold good, such that the product of two rationals or of a rational and an irrational is the same as in the ordinary system, but such that the product of two irrationals is different from their product in the ordinary system. The new definition depends on the well-ordering of the continuum, and an infinity of similar definitions may be set up. The resulting number systems have applications in the foundations of geometry.

26. Denote the fixed quadratic surface by $Q$, its equation by $Q(x)=0$, the polar form of $Q(x)$ by $Q(x, y)$, and the general bilinear form by $B(x, y)$; and write the equations

$$
\begin{aligned}
& \text { (1) } Q(x)=0, \quad \text { (2) } Q(y)=0 \text {, } \\
& \text { (3) } \quad R(x, y, \lambda) \equiv \lambda Q(x, y)+B(x, y)=0 \text {. }
\end{aligned}
$$

Since the region of definition of the variables is limited to $Q$ by (1) and (2), the equations (1), (2), and (3) considered together set up on $Q$ a family of point-conic correspondences which are denoted by $\Gamma(x, y, \lambda)$.

For a fixed value of $\lambda$, to any two consecutive points on $Q$ there correspond by $\Gamma(x, y, \lambda)$ two consecutive conics on $Q$; the two consecutive points determine a line element on $Q$ and the two consecutive conics determine in general two line elements on $Q$. As $\lambda$ varies, $\Gamma(x, y, \lambda)$ establishes a system of contact transformations on $Q$ which are denoted by $T(\lambda)$.

In this paper, Dr. Burgess proves the following: Provided $B(x, y)=0$ is not a null system, a necessary condition that $T(\lambda)$ be a group is that the $\lambda$-matrix of $\lambda Q(x, y)+B(x y)$ possess one of the following characteristics: [1(111)], $\left[1^{\circ}(111)\right]$, $\left[1\left(1^{\circ} 1^{\circ} 1^{\circ}\right)\right],[(211)],\left[\left(2^{\circ} 1^{\circ} 1^{\circ}\right)\right]$.

27. For making a recitation schedule Professor Bowden uses several relation and operation tables, of which the principal ones are (1) a sequence card combining the hours of the week 
into sequences, (2) a course table showing what teachers give and what pupils wish to take the various courses, (3) a conflict table showing the common members of each pair of courses, if their number is not more than a given number, say five, and, if more, showing that fact. The first of these tables is a unary operation table, the second a dyadic relation table, the third a combination of a binary operation table and a dyadic relation table.

The course table is cut into strips showing the class lists, which are used to form the conflict table. This table is then cut into strips, one for each course, which are then easily assembled into groups, so as to have as few conflicts as possible. The courses of any one group are placed at the same sequence of hours.

The form in which the schedule first appears is itself a dyadic relation table, the left-hand column containing the department names, the top row containing the numbers of the sequences, and the intersections of rows and columns the course numbers.

F. N. Cole, Secretary.

\section{A NECESSARY AND SUFFICIENT CONDITION FOR THE UNIFORM CONVERGENCE OF A CERTAIN CLASS OF INFINITE SERIES.}

BY DR. N. J. LENNES.

(Read before the American Mathematical Society, February 25, 1911.)

ALl numbers $x$ such that $a \leqq x \leqq b$ constitute the interval $a b$. A function $f(x)$ is said to be continuous on an interval $a b$ if (1) it is continuous in the ordinary sense for every value of $x, a<x<b,(2)$ if it has right hand continuity for $x=a$ and left hand continuity for $x=b$.

TheOREM. If on the interval $a b$

$$
\sum_{n=0}^{\infty} U_{n}(x)=f(x),
$$

$U_{i}(x)(i=0, \cdots, \infty)$ and $f(x)$ being continuous on the interval $a b$, then in order that $\sum_{n=0}^{\infty} U_{n}(x)$ shall be uniformly convergent on 